\title{
Effect of stroke screening survey on intravenous thrombolysis and long-term outcomes in acute ischemic stroke patients: the real- world evidence from Shanghai, China
}

\author{
Fen $\mathrm{Li}^{1 \# \wedge}$, Yuqian Chen ${ }^{1 \#}$, Lan Hong ${ }^{2}$, Bifan $\mathrm{Zhu}^{1} \wedge$, Duo Chen ${ }^{1}$, Xinyu Qin ${ }^{1}$, Guangfeng Gao ${ }^{3}$, \\ Jianhua $\mathrm{Xu}^{4}$, Xin Cheng ${ }^{2}$, Qiang Dong ${ }^{2,5,6}$, Chunlin Jin ${ }^{1 \wedge}$, Kun Fang ${ }^{2,5,6 \wedge}$
}

${ }^{1}$ Department of Health Policy Research, Shanghai Health Development Research Center, Shanghai, China; ${ }^{2}$ Department of Neurology, Huashan Hospital Affiliated to Fudan University, Shanghai, China; ${ }^{3}$ Jiading Information Center for Health, Shanghai, China; ${ }^{4}$ Department of Neurology, Jiading Central Hospital Affiliated to Shanghai University of Medicine and Health Science, Shanghai, China; ${ }^{5}$ National Center for Neurological Disorders, Shanghai, China; ${ }^{6}$ Shanghai Stroke Service Center, Shanghai, China

Contributions: (I) Conception and design: F Li, Y Chen, K Fang; (II) Administrative support: C Jin, Q Dong; (III) Provision of study materials or patients: G Gao, J Xu, X Cheng; (IV) Collection and assembly of data: F Li, K Fang; (V) Data analysis and interpretation: Y Chen, F Li; (VI) Manuscript writing: All authors; (VII) Final approval of manuscript: All authors.

\#These authors contributed equally to this work.

Correspondence to: Kun Fang, MD, PhD, Department of Neurology, Huashan Hospital Affiliated to Fudan University, No. 12 Middle Wulumuqi Road, Jing'an District, Shanghai 200040, China; National Center for Neurological Disorders, No. 12 Middle Wulumuqi Road, Jing'an District, Shanghai 200040, China; Shanghai Stroke Service Center, No. 12 Middle Wulumuqi Road, Jing'an District, Shanghai 200040, China; Email: drfrankhs@hotmail.com; Chunlin Jin, PhD. International Healthcare PhD, Shanghai Health Development Research Center, No. 1477 West Beijing Road, Jing'an District, Shanghai 200041, China. Email: jinchunlin@shdrc.org.

\begin{abstract}
Background: The stroke screening survey (SSS) is an essential strategy for stroke prevention. However, previous studies rarely discussed the effect of SSS on the acute phase treatment procedure for acute ischemic stroke (AIS) and the long-term prognosis outcomes. This study aims to investigate the effect of SSS on intravenous thrombolysis and long-term outcomes in AIS patients.

Methods: The stroke patients included were collected from Jiading Residences Community Health Records and Shanghai Stroke Service System database, from January 2017 to December 2019. Patients were divided into two groups, according to whether they have been screened before the event (onset and death). Demographic characteristics and treatment information of patients in the two groups were compared by the Mann-Whitney test and Chi-square test. The demographic differences between groups were adjusted with Propensity Score Matching (PSM) to evaluate the effect of SSS on door-to-needle time (DNT). The Kaplan-Meier survival curve with a log-rank test and multiple Cox regression model were used to evaluate the effect of SSS on long-term lifetime.

Results: A total of 1,236 patients with AIS were collected, including 468 (37.86\%) female, 126 (10.19\%) patients with intravenous thrombolysis, 241 (23.30\%) patients died from all-cause mortality by January 8 , 2020. A total of 124 (10.03\%) patients have been screened before AIS onset, and 261 (21.17\%) patients had undergone SSS after AIS onset. The baseline information indicated that patients with previous screening were older than the patients without at the time of onset $[75(70,83)$ vs. $73(65,82), \mathrm{P}=0.017]$, as well as more likely to have a history of hypertension $(90.32 \%$ vs. $78.51 \%, \mathrm{P}=0.002)$ and diabetes $(50.00 \%$ vs. $25.81 \%, \mathrm{P}<0.001)$. PSM results showed that patients with previous screening were associated with less severe onset situation [3 (1, 9) vs. $3(1,5), \mathrm{P}=0.001]$ and shorter DNT [30 (24, 49) vs. $44(31.5,49), \mathrm{P}=0.037]$ when compared to patients without. Additionally, patients with SSS had a lower hazard ratio of 0.567 (95\% CI: $0.380-0.847, \mathrm{P}=0.006$ ) on all-cause mortality.
\end{abstract}

$\wedge$ ORCID: Fen Li, 0000-0002-4081-1970; Yuqian Chen, 0000-0003-1032-0991; Bifan Zhu, 0000-0002-1468-1252; Chunlin Jin, 0000-00034496-9166; Kun Fang, 0000-0002-0476-6842. 
Conclusions: For AIS patients, the SSS is associated with less severe onset situation, shorter DNT, and longer long-term lifetime.

Keywords: Acute ischemic stroke (AIS); intravenous thrombolysis; door-to-needle time (DNT); stroke screening survey (SSS); survival analysis

Submitted Apr 19, 2021. Accepted for publication Jul 26, 2021. doi: 10.21037/atm-21-1971

View this article at: https://dx.doi.org/10.21037/atm-21-1971

\section{Introduction}

Cerebrovascular disease was the second global leading cause of total years of life lost in $2016(1,2)$. The age-standardized incidence was 246.8/100,000 person-years (3). Stroke has risen to be the first cause of death and years of life lost in China (4). In 2018, the death rate for cerebrovascular diseases in China was 149.49 per 100,000 persons, accounting for 1.57 million deaths, and was ranked among the leading causes of death, just behind malignant tumors and heart disease (5). In recent years, the incidence of stroke events has increased due to an aging population (2).

Ten potentially modifiable risk factors, including a history of hypertension and diabetes mellitus, are associated with about $90 \%$ of the attributable risks for stroke, and are especially significant for ischemic stroke $(6,7)$. Therefore, intervening with risk factors effectively is key to the prevention of stroke onset. It has been acknowledged that stroke screening programs and unified community education criteria are essential strategies to promote adherence to stroke prevention $(8,9)$. The stroke screening survey (SSS) is a community-based stroke surveillance program that was established in 2013 in China to promote stroke prevention among residents through a structured face-to-face questionnaire that obtains information about the presence of risk factors and stroke history (10).

The guidelines recommend that intravenous thrombolysis with tissue plasminogen activator (tPA) improves the clinical outcomes of acute ischemic stroke (AIS) (11). Specifically, it has been demonstrated that even when treated within the 4.5-hour time window, the beneficial effect can still decrease over time (12-14). Additionally, the guidelines also recommend that stroke centers should complete the clinical and imaging evaluation and commence intravenous tPA therapy within 60 minutes after AIS patients' arrival (11). This time span is termed the "door-to-needle time" (DNT), which means the time between hospital arrival and administration of intravenous thrombolytics. However, the rate of thrombolysis therapy in China was at a low level. Since most of the acute stroke studies were not primarily designed to assess the effect of SSS on thrombolysis therapy, it is not clear whether SSS can influence the DNT time and long-term survival of patients with AIS.

Thus, in this study, we collected and analyzed the data from Jiading Residences Community Health Records and Shanghai Stroke Service System (4S) database to investigate the effect of SSS on onset age, onset situation, and treatment procedures that include intravenous thrombolysis, as well as long-term outcomes in patients from regional Shanghai with AIS.

We present the following article in accordance with the STROBE reporting checklist (available at https://dx.doi. org/10.21037/atm-21-1971).

\section{Methods}

\section{Patients selected}

We retrospectively reviewed residences with stroke center admission by Residences Community Health Records and the 4S database, from January 2017 to December 2019 in Jiading district, Shanghai, China. Jiading district was chosen for two reasons: firstly, Jiading is a suburban district of Shanghai, which means the situation of this district was relatively stable, with 1.60 million residences at the end of 2019 and there were more elderly people in Jiading (15); secondly, the medical information system in Jiading was constructed better than many other districts in Shanghai, where its electronic health records are helpful to identify patient stroke screening information (16).

The death information for all causes of stroke in these patients was also extracted from the Residences Community Health Records. The patient characteristics, including sociodemographic information (age, sex, race/ethnicity, weight, height, province, address, stroke onset time and 
arrival time, transport to the hospital, route to the hospital, etc.), comorbidities (present-on-admission indicators), and treatment information for the admission were collected from the $4 \mathrm{~S}$. The $4 \mathrm{~S}$ database was established in 2011 as a regional network for stroke care quality improvement that is based on the $4 \mathrm{~S}$ program. This database covers all stroke centers in Shanghai, and all centers document the stroke care process of each patient with the same standardized, customized, and structured electronic record system (17).

Patients with incomplete/wrong unique identification codes or clinical data, such as age, gender, the time on admission, thrombolysis treatment, etc., were also excluded.

\section{SSS in Fiading}

Starting from 2017, the Jiading District, Shanghai, carried out the SSS program, which was able to screen the stroke risk factors in community residents, aged 35 and over (younger than the national version, which has an age threshold of 40) in a voluntary manner to identify high-risk group for stroke. All participants were initially screened using a structured face-to-face questionnaire to obtain information about stroke history, demographic information, and the presence of risk factors. High-risk individuals were identified from the participants according to the main screening factors: whether residents had a genetic history of stroke, and whether they had a history of hypertension, diabetes, atrial fibrillation (AF), hyperlipidemia and smoking, overweight (BMI >25), as well as a lack of physical exercise. Participants with at least 3 risk factors or stroke/transient ischemic attack (TIA) history were defined as high risk. The aim of SSS in Jiading is to improve the predictive accuracy of the stroke screening method. As a reference, the National Risk Assessment Form for HighRisk Stroke Population Screening and Intervention Projects can be found in the appendix, both for the original Chinese version (Supplementary file 1, available online: https:// cdn.amegroups.cn/static/public/10.21037atm-21-1971-1. pdf) and the English translation version (Supplementary file 2, available online: https://cdn.amegroups.cn/static/ public/10.21037atm-21-1971-2.pdf).

\section{Outcome measures and the control variables selected}

In this study, all AIS patients were divided into groups: previous SSS group (had undergone the SSS before AIS onset) and non-previous SSS group (had not undergone the SSS before AIS onset) to evaluate the effect of SSS on the treatment procedure of AIS patients; as well as screened (had undergone the SSS at any time) and non-screened (had not undergone the SSS at any time), to investigate the effect of the SSS on the long-term lifetime.

The demographic characteristics of AIS patients, including age, gender, overweight $(\mathrm{BMI}>25)$, stroke family history, as well as a history of drinking alcohol, smoking, cerebral infarction, hypertension, diabetes, $\mathrm{AF}$ and dyslipidemia were collected to describe the baseline. DNT, National Institutes of Health Stroke Scale (NIHSS), onset-to-door time (ODT), and the thrombolysis ratio were used to compare the effect of SSS on AIS patients with and without previous SSS. Right censoring lifetime after discharge was calculated up to January 8, 2020.

In order to control the confounding effects in the following analysis, personal characteristics and other stroke risk factors that may contribute to the severity and outcomes of stroke events were taken into the analysis as covariates (CVs) with dummy variables, including age, gender, overweight (BMI >25), stroke family history, as well as a history of drinking alcohol, smoking, hypertension, diabetes, AF and dyslipidemia (18).

\section{Statistical analysis}

The statistical analysis was performed through Stata 16 (StataCorp., Ltd, College Station, Texas). Twotailed $\mathrm{P}<0.05$ was considered significant. The baseline demographic and clinical data between patients with or without previous screening were compared. Continuous variables were described as mean and standard deviation (SD) or median and interquartile range (IQR) according to their normality. Normality was tested using the ShaprioWilk test. Differences in demographic and clinical data were compared using Student's $t$-test or Mann-Whitney test for continuous variables, and Chi-square test or Fisher exact test for categorical variables.

A Propensity Score Matching (PSM) analysis was then applied to balance the demographic differences between patients with or without previous stroke screening. To make a trade-off between biases and variance, we chose one-to-four with replacement matching (19). Patients were matched by the CVs mentioned above.

The effect of stroke screening on the survival lifetime of stroke patients was analyzed by the survival analysis model. The death of a patient was regarded as a failure event. The survival period began at the date of discharge for each patient and was right-censored since the death data was updated to January 8, 2020. Kaplan-Meier survival curves 


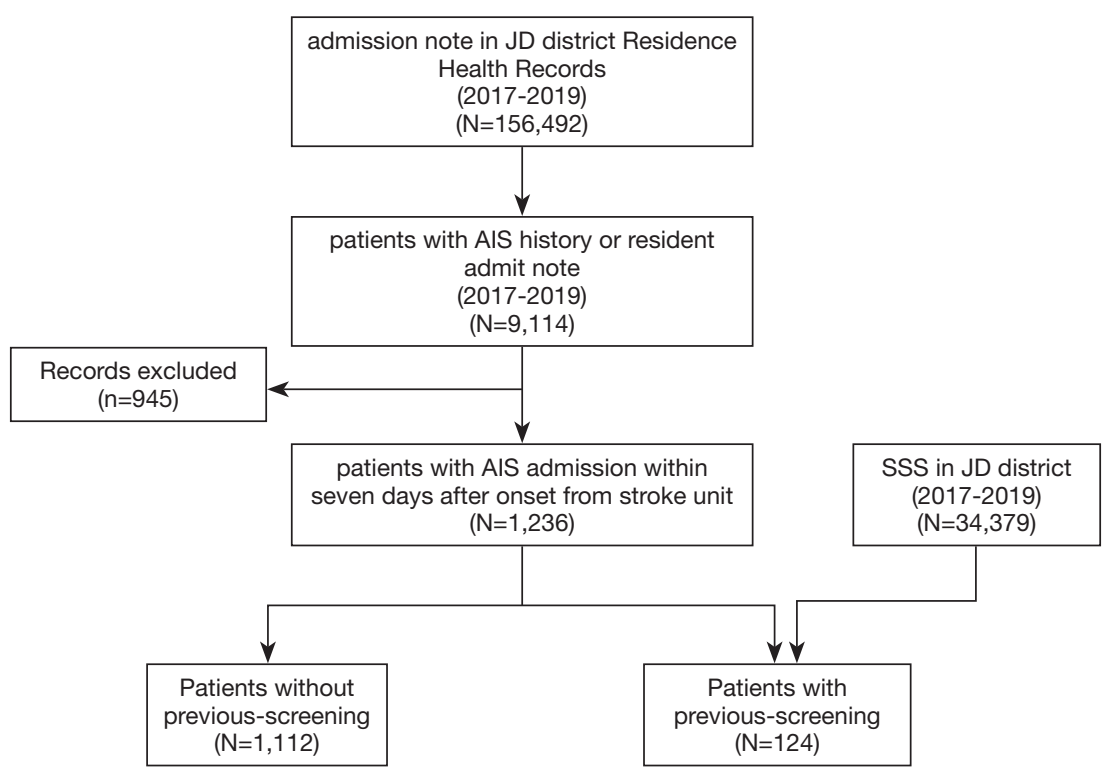

Figure 1 Flow diagram of participation.

were drawn using the log-rank test to test the equality of survivor functions between patients with or without screening before the stroke event and patients with or without screening during the study time (before or after the stroke events). Multivariate Cox regression was applied to analyze the effects of $\mathrm{CVs}$ on the survival of stroke patients. Hazard ratios adjusted for the CVs and corresponding $95 \%$ CIs are provided.

\section{Ethical declarations}

The study was conducted in accordance with the Declaration of Helsinki (as revised in 2013). This study protocol was reviewed and approved by the Ethics Committee of the Shanghai Health Development Research Center (No. 2018025A). The need for written patient consent was waived because of the observational nature of this study.

\section{Results}

\section{Baseline characteristics}

According to the Jiading community health recording system, there were 34,379 persons ( 37,753 times) surveyed with the SSS and 9,114 patients with stroke admission from January 2017 to December 2019. A total of 1,236 patients were selected from the database of 9,114 patients according to AIS admission within seven days after onset and complete demographic characteristics, for final inclusion in this study. Participation at each stage is shown in a flow diagram (Figure 1).

From the 1,236 AIS patients, $124(10.03 \%)$ patients had undergone the SSS before AIS onset, 261 (21.17\%) patients had undergone the SSS after AIS onset. The median onset age of these pre-screened patients was 75 years (IQR 70 83 years), and 51 (41.13\%) were female. Furthermore, 241 $(23.30 \%)$ patients died from all-cause mortality by January 8,2020 , including 30 patients who died in stroke centers. Patients surveyed with the SSS before AIS onset were significantly older than patients without the SSS before AIS [73 (65-82); $\mathrm{P}<0.05$ ], with a much higher percentage of the history of hypertension $(90.32 \%$ vs. $78.51 \%)$ and diabetes mellitus (50.00\% vs. 25.81\%; Table 1). Thus, we should take those indicators under control to explore the effect of stroke screening on stroke treatment. After adjusting the bias of demographic characteristics by PSM, those demographic characteristics in the previous SSS group and non-previous SSS group were balanced $(\mathrm{P}>0.1$; Table 1$)$.

\section{The effect of previous SSS on the procedure of AIS}

For the 126 patients undergoing intravenous thrombolysis, the DNT (30 min; IQR, 24-49 min) of patients previously screened before stroke onset was significantly shorter than the patients who were not previously screened 
Table 1 The comparison of baseline demographic characteristics between the non-previous SSS group and previous SSS group

\begin{tabular}{|c|c|c|c|c|c|c|}
\hline Demographic characteristics & \multicolumn{3}{|c|}{ Overall data } & \multicolumn{3}{|c|}{ Propensity matched } \\
\hline Female, n (\%) & $417(37.50)$ & $51(41.13)$ & 0.429 & $222(44.76)$ & $204(41.13)$ & 0.248 \\
\hline Never smoke & $800(71.94)$ & 85 (68.55) & 0.427 & $342(68.95)$ & $340(68.55)$ & 0.891 \\
\hline Previous smoke & $66(5.94)$ & $10(8.06)$ & 0.349 & $35(7.06)$ & $40(8.06)$ & 0.548 \\
\hline Current smoke & $246(22.12)$ & 29 (23.39) & 0.748 & 119 (23.99) & $116(23.39)$ & 0.823 \\
\hline History of AF, n (\%) & $101(9.08)$ & $10(8.06)$ & 0.707 & $54(10.89)$ & $40(8.06)$ & 0.129 \\
\hline History of dyslipidemia, n (\%) & $20(1.80)$ & $4(3.23)$ & 0.275 & $19(3.83)$ & $16(3.23)$ & 0.606 \\
\hline Overweight, n (\%) & 339 (30.49) & $47(37.90)$ & 0.091 & $204(41.13)$ & $188(37.90)$ & 0.299 \\
\hline Family history of stroke, n (\%) & $16(1.44)$ & $1(0.81)$ & 0.566 & $10(2.02)$ & $4(0.81)$ & 0.106 \\
\hline
\end{tabular}

a , observations used; ${ }^{b}$, patients used. Some patients in the non-previous SSS group were reused due to the one-to-four with replacement matching method. SSS, stroke screening survey; IQR, interquartile range; AF, atrial fibrillation or valvular heart disease; overweight, BMI >25.

[30 (24-49) vs. 40 (30-60) min]. After PSM, 370 patients in the non-previously screened group were matched 4:1 to the previously screened group, and 293 patients were matched once, 49 patients were matched twice, and 28 patients were matched at least 3 times. The baseline characteristics of these matched two groups are listed in Table 2. Previously screened patients had longer ODT compared to their matched counterparts $(\mathrm{P}=0.010)$.

The baseline NIHSS of previous screened patients on admission $[3(1,5)]$ is sightly lower than the matched nonpreviously screened ones $[3(1,9), \mathrm{P}=0.001]$. Specifically, for the patients treated by thrombolysis, patients with previous screening were associated with longer ODT [78 $(44,128)]$ and shorter DNT $[30(24,49)]$ compared to the patients without previous screening [ODT: $67.5(60,100)$; DNT 44 $(31.5,49), \mathrm{P}<0.05$; Table 2).

\section{The effect of SSS on the survival of patients}

Among the 1,236 patients included, 385 (15\%) patients had been screened before or after the stroke onset. The survival possibility for screened patients was significantly longer than the non-screened patients (log-rank test $\mathrm{P}=0.000)$ (Figure 2A). However, there was no significant difference in the survival possibilities between the previous SSS group and non-previous SSS group (Figure 2B). The effects can be justified by the cox regressions. The adjusted hazard ratio of screened patients was 0.567 (95\% CI: $0.380-0.847$, $\mathrm{P}=0.006$ ) after adjusting for confounders (Figure $3 A$ ), while the hazard ratio of the previously screened patients was not significant according to the coefficient shown in Figure $3 B$.

\section{Discussion}

Although the SSS is not designed to improve the therapeutic procedure, we found that the SSS before AIS was associated with shorter DNT in patients with intravenous thrombolysis. Meanwhile, the SSS at any time (before or after stroke onset) was associated with a longer survival time than patients who never had SSS. It reminded us that secondary prevention is also vital for AIS patients. For the prevention effect, it is demonstrated that the SSS was associated with an older age of stroke onset and a lower admission NIHSS score. 
Table 2 The comparison of treatment characteristics between the non-previous SSS group and previous SSS group

\begin{tabular}{|c|c|c|c|c|c|c|}
\hline Treatment characteristics & \multicolumn{3}{|c|}{ Overall data } & \multicolumn{3}{|c|}{ Propensity matched } \\
\hline ODT (IQR) & $300(90,1,440)$ & $532(119,1,787.5)$ & 0.089 & $290(110,1,436)$ & $532(119,1,787.5)$ & 0.010 \\
\hline Admission NIHSS (IQR) & $3(1,8)$ & $3(1,5)$ & 0.102 & $3(1,9)$ & $3(1,5)$ & 0.001 \\
\hline Admission NIHSS $(I Q R)^{c}$ & $6(2,12)$ & $3(1,10)$ & 0.1787 & $7(2,12)$ & $3(1,10)$ & 0.384 \\
\hline DNT $(I Q R)^{c}$ & $40(30,60)$ & $30(24,49)$ & 0.014 & $44(31.5,49)$ & $30(24,49)$ & 0.037 \\
\hline
\end{tabular}

All transfer time was expressed in minutes. ${ }^{a}$, observations used; ${ }^{b}$, patients used. Some patients in the non-previous SSS group were reused due to the one-to-four with replacement matching method; ${ }^{\circ}$, just for patients experienced thrombolytic therapy. SSS, stroke screening survey; IQR, interquartile range; NIHSS, National Institutes of Health Stroke Scale; ODT, onset-to-door time; DNT, door-toneedle time.
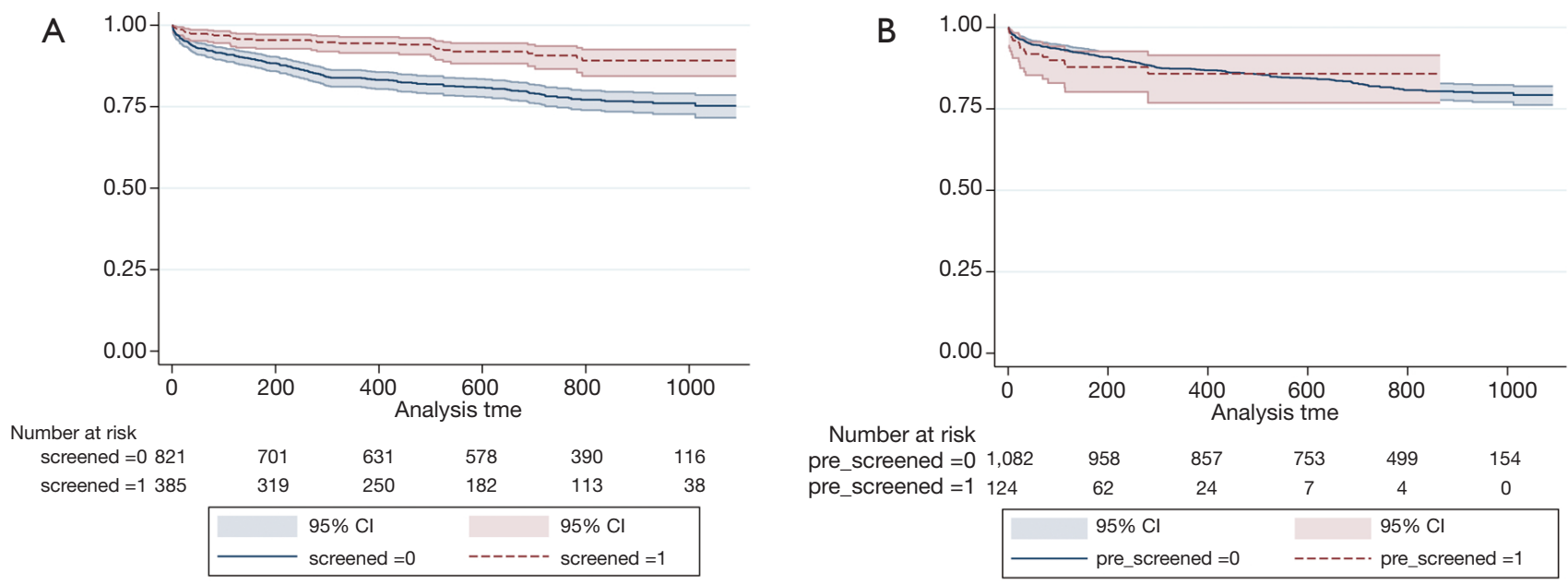

Figure 2 All-cause mortality Kaplan-Meier survival estimates for patients with stroke center admission in Jiading District, from January 2017 to December 2019, by January 8 2020. Patients were divided into two groups according to whether they have done screening by January 8 2020. For each analysis day, the number of people at risk is listed below. The $y$-axis indicates the probability of survival (\%), and the $\mathrm{x}$-axis indicates the survival days since discharge.

The SSS, which is carried out by the general practitioner (GP) in the community health service centers, has been part of the Shanghai Stroke Service System program since 2017 in the Jiading District. The patients who received the SSS before a stroke event appeared to be older and showed higher percentages for the history of hypertension and diabetes mellitus. Given that hypertension and diabetes are critical high-risk factors for stroke $(6,7)$, this finding suggests that populations who had a higher risk of stroke were paid more attention by GPs according to the SSS.
Consequently, among the 34,379 persons (37,753 times) surveyed with the SSS, there were 9,114 patients with stroke admission from January 1, 2017 to December 31, 2019. This provides convincing proof for the effectiveness of the SSS in China.

The first finding of our study was that the SSS might postpone the age of stroke onset and lower admission NIHSS score. After adjusting the confounding factors with PSM, we noticed that the average age of stroke onset for patients who received the SSS was 3 years older than 
A

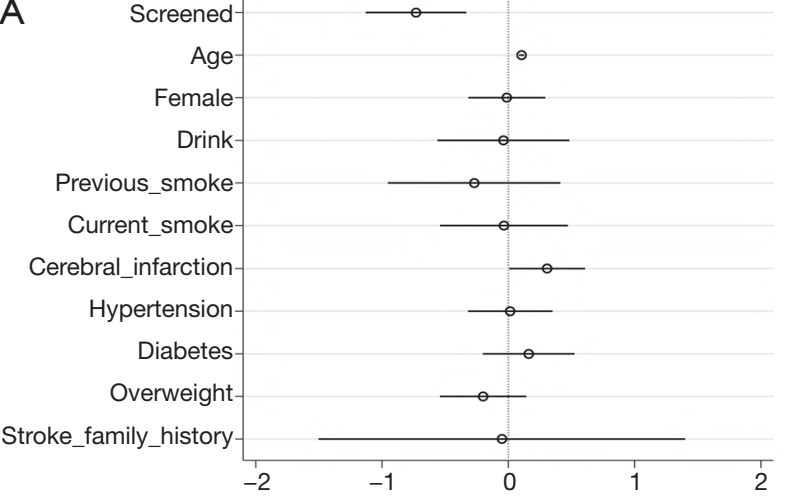

B

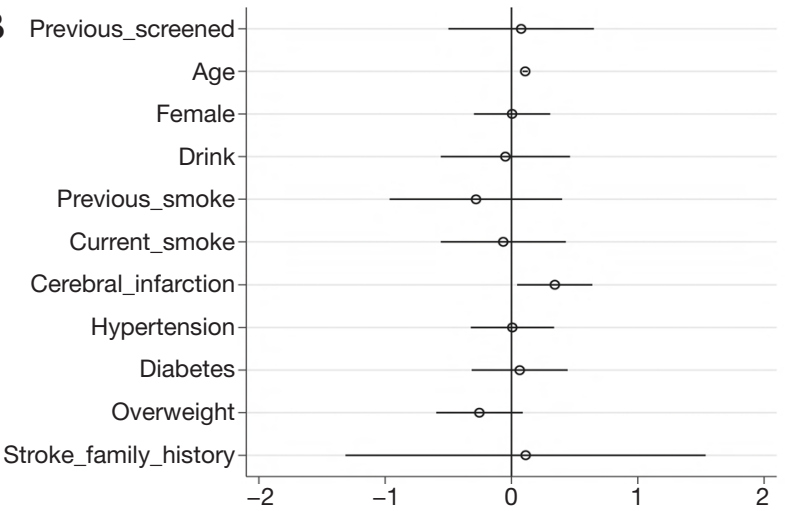

Figure 3 Multivariate Cox regression coefficients to analyze the community-screening effect on the lifetime of stroke patients in Jiading District, from 2017 to 2019. On the left, previous-screened means patients have been screened before the stroke onset in (A). On the right, screened means the patients have been screened before or after the stoke onset. The coefficient of each variable is shown as a hollow dot. $95 \%$ confidence intervals are drawn by the line cross the dot in the figure. Never smoke is taken as baseline. According to the multiregression, unfortunate, screening before onset is not significantly associated with the lifetime of the patients. However, (B) shows that even for people who suffered stroke beforehand, screening could reduce the risk of death.

patients without the SSS, and the effect analysis on the severity of onset showed that the admission NIHSS score for patients who received the SSS was lower than for patients without the SSS. We reasoned that the SSS may be able to change the lifestyle by identifying high-risk factors and promoting follow-up visits. Consistent with the conclusions in our study, a previous study by Barbanti and colleagues, also found that prevention and intervention measures could postpone the onset of cardiovascular, as well as cerebrovascular diseases and reduce their severity (20).

Another key finding in our study was that the DNT in AIS patients with a previous SSS is significantly shorter than in AIS patients without previous SSS. The trend in the relatively high rate of intravenous thrombolysis in AIS patients may be related to a previous SSS, although this was not significant. Though the primary purpose of the SSS was to identify a highrisk group for stroke, health education on stroke identification and treatment methods, such as intravenous thrombolysis, was also carried out by the GPs (21). Therefore, stroke screening enabled patients to have a better understanding of the effectiveness of reperfusion treatment, resulting in a shorter decision time $(22,23)$.

Individuals that were previously screened had a longer ODT, even for the patients treated with thrombolysis, which might be explained by a lower admission NIHSS score in this group. A mild symptom may delay the arrival of patients in Jiading District. Previous studies have shown that patients with TIA or minor stroke can be treated within 24 hours after the onset of symptoms. Moreover, they did not have to be treated by thrombolysis within 4.5 hours $(24,25)$. Patients with previous screening experiences might be told about this information. However, once the patients with previous screening did need to be treated by thrombolysis, they made the decision quicker. That is the ODT is shorter for those patients, as shown in Table 2.

As for the long-term lifetime after stroke onset, it has been widely recognized that prevention and intervention have positive effects on the overall health outcome of stroke patients $(18,26)$. Previous studies generally deem that stroke screening can reduce stroke morbidity and relieve the severity of stroke though dealing with the risk factors of stroke in an effective manner $(6,7)$. However, this study showed that even in stroke patients who were screened after onset, the SSS was associated with a longer lifetime. For the sample included in this study, patients with previous screening were more likely to be older in age, as well as have a history of diabetes, and hypertension, but were also more likely to have a longer lifetime. In this study, the SSS was significantly associated with the treatment regimen, such as thrombolysis. It is widely believed that thrombolysis is essential to the clinical outcome of AIS (11). Lifetime was not significantly different between patients with previous screening before stroke onset and those without. This finding may be due to the limited follow-up time window; therefore, the beneficial effects of SSS on stroke patients might be further proved in future study with a longer 
follow-up period.

In our study, the SSS had no significant effect on the survival time of patients. Of relevance, some regions in Canada introduced an integrated stroke system in the 2009/2010 fiscal year. Evaluation indicated that facilities in provinces with such a system were more likely to care for patients on a stroke unit, as well as have timely access to stroke prevention clinics and tele-stroke services. The reduction in mortality after stroke in provinces with an integrated stroke system may be due to these tendencies, and our results are inconsistent with this possibility (27). One reason may be that there is a process from knowledge to action, and the SSS could improve stroke-related knowledge (9). Nevertheless, a study of 187,723 adults in 2017 showed that even if patients had correct knowledge of stroke and its symptoms, many patients could not make correct behavioral decisions that were timely upon when stroke onset (28). Another reason could be that patient with a SSS had a lower admission NIHSS score and benefited less from hospital treatment. This is in accord with the marginal decrease in effect.

However, our study also found that AIS patients who had the SSS at any time, whether before or after the incident of stroke, may have a longer survival time. This indicates that the SSS is still essential for patients with a history of AIS, which may be due to good secondary prevention conducted by the SSS after the initial event. The SSS should not be limited to the prevention roles in controlling stroke controlling. Furthermore, the SSS should pay more attention to collect sensitize on more information about stroke treatment, like thrombolysis time window, the alternative therapeutic regimens for different types of patients, which is expected and the key points in the convalescence and after discharging, to improve the health status of patients with a history of stroke.

\section{Strength and limitations}

As far as we know, this is the first paper to focus on the absolute effect on people who experienced early stroke prevention, like the SSS. This is a large retrospective cohort study based on real-world data. The data from this project mainly comes from the Residences Community Health Records, which can cover all residents in the pilot area and can provide precise screening information, treatment behaviors and health outcomes. Thus, the findings in this study could provide an evidence-based suggestion for the improvement of the SSS. However, the validity of the result from this study results was limited by the quality of the data. Given that this is an observational retrospective study, there are considerations that should be made. Firstly, even though the PSM method was applied to adjust for potentially confounding factors, there were some inevitable differences and biases in the demographic and clinical data. Secondly, due to the limited sample size, further analysis to investigate the influence of the SSS on the decision-making of intravenous thrombolysis in AIS patients within the intravenous thrombolysis time window could not be carried out. Thirdly, due to the quality of the real-world data involved in this study, with an extensive amount of missing data, and relatively less variation, we could not conduct further empirical analysis that evaluates a wider range of clinical outcomes in this study. However, we are planning to implement this in the future by collecting more and high-quality data. Fourthly, related to the minor stroke onset situation of the sample in this study, the effect of the SSS on those patients with minor stroke was less reliable than on patients with more severe stroke (NIHSS >3). Further investigations should be done to extend the findings in this study.

\section{Conclusions}

The SSS before AIS was associated with shorter DNT in AIS patients with intravenous thrombolysis. The SSS at any time (before or after stroke onset) was associated with a longer survival time than patients that never had the SSS. These findings suggest that the SSS plays a vital role in the whole course of stroke, not just prevention.

\section{Acknowledgments}

We thank the reviewer for the helpful and constructive comments on our work.

Funding: The research design was funded by and based on the Outstanding Academic Leader Program and the Outstanding Young Medical Personnel Training Program of the Shanghai Municipal Health Commission (Grant No. 2018YQ51) and National Natural Science Funds of China' Empirical Study on Evaluation of Integrated Stroke Prevention and Treatment System Based on Real-World Data (Grant No. 72004138).

\section{Footnote}

Reporting Checklist: The authors have completed the 
STROBE reporting checklist. Available at https://dx.doi. org/10.21037/atm-21-1971

Data Sharing Statement: Available at https://dx.doi. org/10.21037/atm-21-1971

Peer Review File: Available at https://dx.doi.org/10.21037/ atm-21-1971

Conflicts of Interest: All authors have completed the ICMJE uniform disclosure form (available at https://dx.doi. org/10.21037/atm-21-1971). The authors have no conflicts of interest to declare.

Ethical Statement: The authors are accountable for all aspects of the work in ensuring that questions related to the accuracy or integrity of any part of the work are appropriately investigated and resolved. The study was conducted in accordance with the Declaration of Helsinki (as revised in 2013). This study protocol was reviewed and approved by the Ethics Committee of the Shanghai Health Development Research Center (No. 2018025A). The need for written patient consent was waived because of the observational nature of this study.

Open Access Statement: This is an Open Access article distributed in accordance with the Creative Commons Attribution-NonCommercial-NoDerivs 4.0 International License (CC BY-NC-ND 4.0), which permits the noncommercial replication and distribution of the article with the strict proviso that no changes or edits are made and the original work is properly cited (including links to both the formal publication through the relevant DOI and the license). See: https://creativecommons.org/licenses/by-nc-nd/4.0/.

\section{References}

1. GBD 2016 Causes of Death Collaborators. Global, regional, and national age-sex specific mortality for 264 causes of death, 1980-2016: a systematic analysis for the Global Burden of Disease Study 2016. Lancet 2017;390:1151-210.

2. Kim J, Thayabaranathan T, Donnan GA, et al. Global Stroke Statistics 2019. Int J Stroke 2020;15:819-38.

3. Wang W, Jiang B, Sun H, et al. Prevalence, Incidence, and Mortality of Stroke in China: Results from a Nationwide Population-Based Survey of 480687 Adults. Circulation 2017;135:759-71.
4. Zhou M, Wang H, Zeng X, et al. Mortality, morbidity, and risk factors in China and its provinces, 1990-2017: a systematic analysis for the Global Burden of Disease Study 2017. Lancet 2019;394:1145-58.

5. Wang YJ, Li ZX, Gu HQ, et al. China Stroke Statistics 2019: A Report From the National Center for Healthcare Quality Management in Neurological Diseases, China National Clinical Research Center for Neurological Diseases, the Chinese Stroke Association, National Center for Chronic and Non-communicable Disease Control and Prevention, Chinese Center for Disease Control and Prevention and Institute for Global Neuroscience and Stroke Collaborations. Stroke Vasc Neurol 2020;5:211-39.

6. O'Donnell MJ, Xavier D, Liu L, et al. Risk factors for ischaemic and intracerebral haemorrhagic stroke in 22 countries (the INTERSTROKE study): a case-control study. Lancet 2010;376:112-23.

7. O'Donnell MJ, Chin SL, Rangarajan S, et al. Global and regional effects of potentially modifiable risk factors associated with acute stroke in 32 countries (INTERSTROKE): a case-control study. Lancet 2016;388:761-75.

8. Gropen T, Magdon-Ismail Z, Day D, et al. Regional implementation of the stroke systems of care model: recommendations of the northeast cerebrovascular consortium. Stroke 2009;40:1793-802.

9. Willoughby DF, Sanders L, Privette A. The impact of a stroke screening program. Public Health Nurs 2001;18:418-23.

10. Guan T, Ma J, Li M, et al. Rapid transitions in the epidemiology of stroke and its risk factors in China from 2002 to 2013. Neurology 2017;89:53-61.

11. Powers WJ, Rabinstein AA, Ackerson T, et al. 2018 Guidelines for the Early Management of Patients With Acute Ischemic Stroke: A Guideline for Healthcare Professionals From the American Heart Association/ American Stroke Association. Stroke 2018;49:e46-e110.

12. Lees KR, Bluhmki E, von Kummer R, et al. Time to treatment with intravenous alteplase and outcome in stroke: an updated pooled analysis of ECASS, ATLANTIS, NINDS, and EPITHET trials. Lancet 2010;375:1695-703.

13. Zheng H, Yang Y, Chen H, et al. Thrombolysis with alteplase 3-4.5 hours after acute ischaemic stroke: the first multicentre, phase III trial in China. Stroke Vasc Neurol 2020;5:285-90.

14. Fonarow GC, Zhao X, Smith EE, et al. Door-to-needle times for tissue plasminogen activator administration and 
clinical outcomes in acute ischemic stroke before and after a quality improvement initiative. JAMA 2014;311:1632-40.

15. Shanghai Bureau of Statistics. 2020. Statistical Bulletin on National Economic and Social Development of Shanghai in 2019. Available online: http://tjj.sh.gov.cn/tjgb/202003 29/05fof4abb2d448a69e4517f6a6448819.html

16. Yang T, Li F, Zhu B, et al. An Exploratory Study of the Use of the Electronic Health Records of Hypertensive Patients to Support the Primary Prevention of Stroke in Shanghai. Risk Manag Healthc Policy 2020;13:1781-9.

17. Dong Y, Fang K, Wang X, et al. The network of Shanghai Stroke Service System (4S): A public health-care webbased database using automatic extraction of electronic medical records. Int J Stroke 2018;13:539-44.

18. Li RC, Xu WD, Lei YL, et al. The risk of stroke and associated risk factors in a health examination population: A cross-sectional study. Medicine (Baltimore) 2019;98:e17218.

19. Stuart EA, Rubin DB. Matching methods for causal inference: Designing observational studies. Harvard University Department of Statistics mimeo, 2004.

20. Barbanti M, Todaro D, Costa G, et al. Optimized Screening of Coronary Artery Disease With Invasive Coronary Angiography and Ad Hoc Percutaneous Coronary Intervention During Transcatheter Aortic Valve Replacement. Circ Cardiovasc Interv 2017;10:e005234.

21. Oostema JA, Carle T, Talia N, et al. Dispatcher Stroke

Cite this article as: Li F, Chen Y, Hong L, Zhu B, Chen D, Qin X, Gao G, Xu J, Cheng X, Dong Q, Jin C, Fang K. Effect of stroke screening survey on intravenous thrombolysis and long-term outcomes in acute ischemic stroke patients: the real-world evidence from Shanghai, China. Ann Transl Med 2021;9(17):1363. doi: 10.21037/atm-21-1971
Recognition Using a Stroke Screening Tool: A Systematic Review. Cerebrovasc Dis 2016;42:370-7.

22. Li C, Lumey LH. Impact of disease screening on awareness and management of hypertension and diabetes between 2011 and 2015: results from the China health and retirement longitudinal study. BMC Public Health 2019;19:421.

23. Brienza RS. At a Crossroads: The Future of Primary Care Education and Practice. Acad Med 2016;91:621-3.

24. Wang Y, Pan Y, Zhao X, et al. Clopidogrel With Aspirin in Acute Minor Stroke or Transient Ischemic Attack (CHANCE) Trial: One-Year Outcomes. Circulation 2015;132:40-6.

25. Wang Y, Wang Y, Zhao X, et al. Clopidogrel with aspirin in acute minor stroke or transient ischemic attack. N Engl J Med 2013;369:11-9.

26. Kivioja R, Pietilä A, Martinez-Majander N, et al. Risk Factors for Early-Onset Ischemic Stroke: A Case-Control Study. J Am Heart Assoc 2018;7:e009774.

27. Ganesh A, Lindsay P, Fang J, et al. Integrated systems of stroke care and reduction in 30-day mortality: A retrospective analysis. Neurology 2016;86:898-904.

28. Li S, Cui LY, Anderson C, et al. Public Awareness of Stroke and the Appropriate Responses in China: A CrossSectional Community-Based Study (FAST-RIGHT) Stroke 2019;50:455-62. 